\title{
Investigating the Effect of Drugs Thiopental Sodium and Propofol in Patients Undergoing Electroconvulsive Therapy, RCT
}

\author{
Hamid Kayalha ${ }^{1}$, Asghar Karbord ${ }^{2}$, Mohammad Qasem Roushanfekr ${ }^{3}$, Zahra mozaffari ${ }^{4}$ \\ Marzie Bigom Khezri ${ }^{5}$, \\ ${ }^{1}$ Assistant anesthesiology \& Faculty members of anesthesiology group of medicine Dept. Qazvin \\ University of Medical Sciences, Qazvin, Iran \\ ${ }^{2}$ MSc epidemiologist, faculty members of surgical technologist group of Paramedical Sciences Dept. \\ Qazvin University medical science, Qazvin, Iran \\ ${ }^{3}$ Assistant anesthesiology \& Faculty members of anesthesiology group of medicine Dept. Qazvin \\ University of Medical Sciences, Qazvin, Iran \\ ${ }^{4}$ Undergraduate student in surgical technology of surgical technologist group of Paramedical \\ Sciences Dept. Qazvin University medical science, Qazvin, Iran \\ ${ }^{5}$ Professor of anesthesiology \&Faculty members of anesthesiology group of medicine Dept. Qazvin \\ University of Medical Sciences, Qazvin, Iran
}

*Corresponding Author: Asghar Karbord, MSc epidemiologist, faculty members of surgical technologist group of Paramedical Sciences Dept. Qazvin University medical science, Qazvin, Iran

\begin{abstract}
Background: Electroconvulsive Therapy (ECT) in the treatment of mental illnesses and the importance of using an anesthesia method that causes the least hemodynamic changes in the patient, we decided to evaluate their effects in this case with both thiopental sodium and propofol.

Aim: This study aimed to compare the hemodynamic effects of the drug, sodium thiopental and propofol common among the anesthetic agent may be, in patients treated with ECT is done.

Materials and Methods: This study hospital 22 Bahman in Qazvin on 84 patients in two groups :) 42 patients in thiopental sodium group and 42patients in propofol group) electroconvulsive brain under general anesthesia by propofol and thiopental has been carried out. Hemodynamic changes in systolic and diastolic blood pressure ( $\mathrm{mm} \mathrm{mmHg}$ ) and pulse rate, number of minutes, duration of seizure and recovery time ( $\mathrm{min})$ size were recorded.
\end{abstract}

Results: Measuring blood pressure and pulse before and after anesthesia and ECT show that, in patients in thiopental sodium group changes in systolic blood pressure, diastolic and pulse rate. Respective 45\%and $64 \%$ and $26 \%$ in the third minute of the study. In the propofol group, the highest systolic, diastolic blood pressure changes were $55 \%$ and $64 \%$ at the tenth minute, respectively, but the highest pulse rate increase was at the third minute and 33\%, respectively. Test Friedman is also a significant relationship between consumption of these drugs, blood pressure showed that The duration of seizures during ECT sodium thiopental less than group $B$, but the duration of anesthesia with propofol shorter return is .The results were: Due to better hemodynamic stability of sodium thiopental, propofol, the drug is preferred to more.

Keywords: Cerebral Electroconvulsive, Thiopental Sodium, Propofol

\section{INTRODUCTION}

Brain (electroconvulsive (ECT, electric digital planning system to stimulate the central nervous system to create continuous should be stimulation which is the minimum time for the effectiveness of the electrical stimulation between 25 seconds to one minute. ECT does an important role in the treatment of mental illnesses such as: acute and chronic depression, mania, schizophrenia, suicide attempt, catatonia, and Parkinson's disease (1-3). This electrical stimulation initially triggered generalized tonic activity for 10 min Seconds and then clonic activity is generalized from a few seconds to over a minute (6- 4) .The duration of brain stimulation depends on many factors, including the patient's age, regenerative energy, electrode location, seizure threshold, and prescription drugs(7). Electrical activity first causes parasympathetic stimulation, bradycardia, and hypotension, followed by sympathetic 
stimulation of tachycardia, and hypertension and premature ventricular contraction $(8,9)$. In the clonic phase, the sympathetic system is stimulated and causes tachycardia, elevated blood pressure and PVC, and rarely ventricular tachycardia, which is spontaneously restricted for up to 2 minutes (9). ECG changes are caused by a decrease in the negative ST and $\mathrm{T}$ fragments without an increase in cardiac enzymes, on the other hand, the most common cause of death is ECT following myocardial infarction and cardiac dysrhythmia (10). ECT stimulates the nervous system such as brain working and its effects on the cardiovascular system - cardiovascular and central nervous system (11). finding a drug to induce restful sleep anesthesia and minimal effects on blood pressure and heart rate and recovery quick is one of the main goals of anesthetists are logged in, the application of a suitable drug with the lowest hemodynamic changes and etc. more time in the film shorter lead could be one of the objectives of anesthesia is (12). Methohexital because seizure (duration) compared to Propofol ) and recovery short (compared with thiopental sodium) and less side effects (compared to Etomidate) drug of choice is, if the seizure was long (more than 100 seconds), propofol Methohexitalis preferred, on the other hand propofol because of the decreased responses in blood pressure and heart rate after ECT alternative Methohexital is (13). Electroconvulsive therapy brain works is not fully known, but mental patients who have been spontaneous seizures have decreased mental disease severity, $(3,2)$ may be a biological antagonism between schizophrenia and seizures are present. (3) Therefore, understanding the effects of drugs used for seizures may be in the course of therapy to be effective. Propofol and thiopental sodium, causing an increase in the parameters of hemodynamics beating heart pressure blood systolic is but the increase compared to thiopental more Propofol also need more energy to induce ECT exist and instead the electrical stimulation longer be BUH (10) .

The patients are the cause of depression treated with ECT and three-drug sodium thiopental, propofol and midazolam for induction of anesthesia, which causes the heart rate, systolic blood pressure and diastolic blood pressure to rise and thiopental increase is greater hemodynamic criteria. But propofol has a better recovery (14).According to the results of these papers about of drug; propofol is safe and convenient for ECT, and Methohexital and sodium thiopental routine drug of anesthesia in Iran instead of anesthetists is available and it can be evaluated pharmacological effects in patients with stable best results Hemodynamics and reduction of potential complications are among the tasks of anesthesiologists and psychiatrists (15). To the mines reasons the decision was therefore the time etc. recovery, hemodynamic changes in patients under anesthesia drug, sodium thiopental and propofol to treat patients by ECT to be compared.

\section{MATERIALS AND MethodS}

This study is a clinical trial, double-blind design with the aim of comparing Hemodynamic recovery after anesthesia with 2groupspatients: Group A: drug propofol and group B:drug, sodium thiopental linfused for anesthesia then ECT was done. This study was performed at 22 Bahman Hospital in Qazvin province of Iran with inclusion and exclusion criteria: history of psychiatric hospitalization and expert psychiatrist approval for electroconvulsive therapy, no history of heart disease, no allergy to the studied drugs, ages 15 to 40 years, no pregnancy and no Lactation, no history of chronic diseases and no history of heart and brain surgery were selected in 84 patients. Patients were randomly divided into two groups, group A (Propofol) 42patients and Group B ( Thiopental Sodium)42patients Individuals were divided to induce anesthesia . Before anesthesia, anesthesia class (ASA) patients were identified and questionnaire data were collected on age, sex, fasting patients with blood pressure monitoring and electrocardiography and pulse rate were ready for ECT. Prior to anesthesia, blood pressure and pulse rate were recorded. Induction of anesthesia with $0.5 \mathrm{mg}$ atropine and group $\mathrm{B}$ using thiopental sodium drugs $(2 \mathrm{mg} / \mathrm{kg})$ And the Group Apropofol $(1 \mathrm{mg} / \mathrm{kg})$ In addition, Succinylcholine $(0.5 \mathrm{mg} / \mathrm{kg})$ Carried out one minute after the injection and then Succinylcholine patient blood pressure and pulse rate ECT induced. In the third minute after ECT stimulates the patient's pulse rate and blood pressure measured again was recorded. Recovery time, eye opening to verbal response and keep the head up for 5 seconds stopwatch measurement was recorded. Blood pressure and pulse rate of patient size tenth minute was recorded. At the end, all information about both groups of patients is entered into the software SPSS Version 22 was analyzed and with Statistical tests $\mathrm{T}$ test, Repeated measures Significance level ( 0.05 ) was assessed. 
Investigating the Effect of Drugs Thiopental Sodium and Propofol in Patients Undergoing Electroconvulsive Therapy, RCT

\section{ReSults AND CONCLUSIONS}

In this study of both groups: 50 males and 34 females with a mean $\& S D$ age of the men who were thiopental $(30.2 \pm 13.5)$ and for propofol $(25.7 \pm 7.5)$.Mean $\&$ SD of age of women who anesthesia with thiopental sodiumtobe taken $(40.5 \pm 13.4)$ and for propofol $(20.9 \pm 36.5)$. B group patients were anesthetic with thiopental sodium and A group patients were anesthetic with propofol. The mean blood pressure systolic and diastolic terms $(\mathrm{mmHg})$ before thiopental(126/103) and in the first minute after $(127 / 86)$ and third minuet after induction of anesthesia and having ECT (150/106)and in the tenth minute after induction of anesthesia, seizures equipping (146/101) respectively .

In the third minute after induction of anesthesia and shock, the highest (45\%) changes in systolic blood pressure were observed. The least changes $(22 \%)$ were seen in the first minute after induction of anesthesia. Friedman test also showed a significant relationship between propofol intake and systolic blood pressure changes $(\mathrm{P}<0.001)$. The most $(64 \%)$ of diastolic blood pressure changes were seen at the third minute after induction of anesthesia and ECT. However, the lowest (32\%) diastolic blood pressure changes were in the first minute after induction of anesthesia. Friedman test showed a significant relationship $(<\mathrm{p} 0.001)$ between thiopental sodium intake and diastolic blood pressure changes. Mean blood pressure (systolic and diastolic in millimeters of mercury) before induction with propofol was (97/65).In the first minute after induction of anesthesia and before ECT, (130 / 89)and in the third minute after induction of anesthesia and ECT, (148/ 106)and in the 10th minute after induction of anesthesia findings show that the highest changes in systolic and diastolic blood pressure occurred at induction anesthesia and before the ECT and complete seizure was( 152/ 106). The above

the 10th minute after induction of anesthesia, which showed a 55\% and 64\% increase, respectively, and the smallest changes in systolic and diastolic blood pressure, respectively, at 33\% and $37 \%$, respectively. The first occurred after induction of anesthesia. Friedman test showed a significant relationship $(<\mathrm{p} 0.001)$ between propofol consumption and changes in blood pressure (systolic and diastolic). He mean pulse rate (number per minute) of patients before anesthesia with sodium thiopental was( $85 \pm 17$ ) and at one, three and ten minutes after the start of anesthesia with sodium thiopental was( $97 \pm$ 18),( 107 \pm 28$),(98 \pm 20)$.Also, the highest pulse changes were seen in the third minute after the start of anesthesia with sodium thiopental and ECT and showed a $26 \%$ increase. The smallest changes were seen ten minutes after the start of anesthesia with thiopental sodium, which showed a $15 \%$ increase.

Friedman test showed a significant relationship $(<\mathrm{p} 0.001)$ between thiopental sodium intake and pulse changes. The mean pulse rate of patients before propofol anesthesia was $(85 \pm 19)$ and at one, three and ten minutes after the start of propofol anesthesia were $(107 \pm 21),(113 \pm 25)$ and (101 \pm 19$)$, respectively. Also, most of the pulse changes occurred in the third minute after the start of anesthesia and propofol ECT and showed a $33 \%$ increase. The smallest changes occurred at 10 minutes after the start of propofol anesthesia, showing an increase of about $18 \%$.Friedman test also showed a significant relationship $(<\mathrm{p} 0.001)$ between propofol consumption and pulse changes. The mean duration of electro convulsions with thiopental sodium (s) was $(25 \pm 13)$ and with propofol (53 \pm 28 ). The mean duration of recovery from anesthesia with thiopental sodium was $(13 \pm 5)$ and propofol $(9 \pm 3)$. T-test showed a significant relationship between the use of these two drugs and the duration of ECT and the duration of return from anesthesia.

Table1. Mean and standard deviation of age for men and women in two groups: thiopental sodium and propofol

\begin{tabular}{|c|c|c|}
\hline Propofol & Sodium thiopental & \\
\hline $25 / 7 \pm 7 / 7$ & $30 / 2 \pm 13 / 5$ & Men \\
\hline $20 / 9 \pm 36 / 5$ & $40 / 5 \pm 13 / 4$ & Women \\
\hline
\end{tabular}

Table2. Comparison of mean blood pressure and pulse in two groups of thiopental sodium and propofol

\begin{tabular}{|c|c|c|c|}
\hline $\mathrm{P}$ Value & Propofol & Sodium thiopental & name \\
\hline $\mathrm{P}<0.05$ & $97 / 79 \pm 9 / 716$ & $103 / 78 \pm 13 / 359$ & Anesthesia before systole \\
\hline $\mathrm{P}<0.05$ & $65 / 00 \pm 74 / 00$ & $65 / 12 \pm 8 / 403$ & Diastole before anesthesia \\
\hline $\mathrm{P}<0.05$ & $130 / 47 \pm 11 / 934$ & $127 / 32 \pm 14 / 496$ & First systole minute \\
\hline $\mathrm{P}<0.05$ & $89 / 30 \pm 10 / 997$ & $86 / 59 \pm 13 / 343$ & The first minute of diastole \\
\hline $\mathrm{P}<0.05$ & $148 / 372 \pm 11 / 938$ & $150 / 98 \pm 10 / 910$ & Third minute systole \\
\hline
\end{tabular}


Investigating the Effect of Drugs Thiopental Sodium and Propofol in Patients Undergoing Electroconvulsive Therapy, RCT

\begin{tabular}{|c|c|c|c|}
\hline $\mathrm{P}<0.05$ & $106 / 51 \pm 10 / 885$ & $106 / 59 \pm 11 / 093$ & Third diastole \\
\hline $\mathrm{P}<0.05$ & $152 / 33 \pm 16 / 011$ & $146 / 59 \pm 12 / 155$ & Tenth systole \\
\hline $\mathrm{P}<0.05$ & $106 / 98 \pm 11 / 24$ & $101 / 95 \pm 13 / 457$ & 10 minutes diastolic \\
\hline $\mathrm{P}<0.05$ & $85 / 93 \pm 19 / 230$ & $85 / 02 \pm 16 / 915$ & Anesthesia before pulse \\
\hline $\mathrm{P}<0.05$ & $107 / 47 \pm 21 / 08$ & $97 / 44 \pm 18 / 014$ & The first minute pulse \\
\hline $\mathrm{P}<0.05$ & $113 / 98 \pm 25 / 701$ & $107 / 10 \pm 28 / 729$ & Third minute pulse \\
\hline $\mathrm{P}<0.05$ & $101 / 6 \pm 19 / 605$ & $98 / 05 \pm 20 / 698$ & Tenth minute pulse \\
\hline
\end{tabular}

Table3. Long time, seizures, apnea and recovery

\begin{tabular}{|c|c|c|c|}
\hline P Value & Propofol & Sodium thiopental & name \\
\hline $\mathrm{P}<0.05$ & $9 / 81 \pm 3 / 648$ & $13 / 70 \pm 5 / 103$ & Recovery time( minutes) \\
\hline $\mathrm{P}<0.05$ & $53 / 3 \pm 28 / 6$ & $25 / 7 \pm 13 / 4$ & Seizure duration (seconds) \\
\hline
\end{tabular}

\section{DISCUSSION}

In this study, propofol $(1 \mathrm{mg} / \mathrm{kg})$ was not able to inhibit hemodynamic changes in patients as much as thiopental sodium $(2 \mathrm{mg} / \mathrm{kg})$. But B Bolaji and colleagues in Nigeria in 2012 examined 60 patients who received ECT. They found that propofol (1 $\mathrm{mg} / \mathrm{kg}$ ) more than thiopental sodium (5 $\mathrm{mg} / \mathrm{kg}$ ) prevented diastolic pressure and mean arterial pressure. Propofol also has a faster recovery than thiopental sodium (15).Short recovery time is consistent with our study. Because the effectiveness of cerebral electrocution depends on the duration of the seizure that occurs during electrocution. Therefore, even as long as possible, induction of anesthesia should not adversely affect seizure duration (21).Kumar A concluded that patients taking propofol needed more energy to induce seizures but instead had longer seizures (14). Our research has shown that propofol is a better drug than thiopental sodium because it produces a longer seizure than thiopental sodium. Because brain electrical shock stimulates the autonomic nervous system and most of its effects on the cardiovascular system and the central nervous system, therefore, the use of the appropriate drug that causes the least hemodynamic changes can play an important role in maintaining the health and safety of patients (22).In 2009 Puran Akhavan Akbari and his colleagues studied 16 patients who received 72 ECTs in total. The study found that hemodynamic abnormalities in the group receiving induction of anesthesia were less than the group receiving thiopental sodium for induction of anesthesia. The study found that the duration of seizures did not differ between the two groups (16).in Desai, Rahul, Belgium, in 2009 studied fifty patients who needed ECT. They used thiopental sodium $(4 \mathrm{mg} / \mathrm{kg}$ ) and propofol $(5 \mathrm{mg} / \mathrm{kg}$ ) for induction. The study found that hemodynamic changes were less pronounced in the propofol group (17). The results of this study showed that the duration of return from anesthesia with propofol is shorter than thiopental sodium and therefore propofol is a more appropriate drug (18).

This part of the study is consistent with the Prtibha Jain Shah study, which used three drugs thiopental sodium, propofol and midazolam to induce anesthesia in patients and concluded that propofol anesthesia was faster than the other two drugs. Our study also showed that, despite significant hemodynamic changes, there was no statistically significant difference in prophylactic ECT with two thiopental sodium and propofol drugs, which were more prominent in the propofol group (19).

Instead, propofol produces longer seizures than thiopental sodium, which prolongs the seizure time, leading to faster recovery and greater therapeutic effects for the patient (20).

On the other hand, ECT with Propofol anesthesia results in shortening the recovery time from anesthesia, this represents economical cost savings with this drug because recovery requires manpower and is costly. It is recommended to use a dose higher than (mg / $\mathrm{kg}$ ) for better control of hemodynamic changes if propofol is used.

\section{REFERENCES}

[1] Ronald D.Miller. Millers Anesthesia .Seventh edition. United States of America. 2010. 2445 6, 2477-99

[2] Roberto L. Hines, Katherine E. Marchall. Stoeltings Anesthesia and Co- EXISTING Disease. 2012: 537-8

[3] Benjamin J.Sadock, Virginia Alcott Sadock, Pedro Ruiz Kaplan\&Sadocks Comprehensive text book of psychiatry. Ninth Edition. 2009. 3285-6.

[4] Selvin BL. Electroconvulsive therapy. Anesthesiology 1987; 67: 367.

[5] Ronald D. Miller textbook of anesthesia, 9th ed, Elsevier Churchill Livingstone 2000; PP: 2263-4. 
Investigating the Effect of Drugs Thiopental Sodium and Propofol in Patients Undergoing Electroconvulsive Therapy, RCT

[6] Holden C. A guarded endorsement for shock therapy. Science 1985; 228: 1510.

[7] Swartz CM. Anesthesia for ECT. Convuls Ther 1993; 9: 301.

[8] Mc Call WV. A systole in electro convulsive therapy, Report of four cases. J Clin Psychiatry 1996; 57:199.

[9] Rice EH, Sombroto LB, Markowitz JC, et al. Cardiovascular morbidity in high - risk patients during ECT. AM J Psychiatry 1994; 151: 1637.

[10] Kumar / Alok and et al. A comparison of Propofol and thiopontone for electroconvulsive therapy. Journal of Anesthesiology clinical pharmacology. 2012; 28(3).

[11] Trzepacz PT, Weniger FC, Greenhouse J. Etomidate anesthesia increase seizure duration ECT, A retrospective study, Gen Hosp Psychiatry 1993; 15: 115.

[12] Kay B, Rolly G. IcI 32868, a new intravenous induction agent. Acta Anesthesiol Belg 1977; 23: 303 .

[13] Shah PJ, Dubey KP, Watti C, Lalwani J. Effectiveness of thiopentone, propofol and midazolam as an ideal intravenous anesthetic agent for modified electroconvulsive therapy: A comparative study. Indian 2010; 54(4):296-301. doi: 10.4103/0019-5049.68371.

[14] Nishiyama. M, Togashi. H .Effects of anesthetic agents on seizure duration and hemodynamics inelectrocvulsive therapy. Masui. 2009; 58(10): 1266-9.

[15] Pooran Akhvan akbari , Parviz Molavi, Ghodrat Akhvan akbari Mohammad Reza Ghodrati. Comparing the Effects of Propofol and Sodium Thiopental in the Induction of Anesthesia for Electro Convulsive Therapy (ECT).Journal of Ardabil University of medicine. Number 3 (autumn 2009) Volume9 :123-130
[16] Desai, Rahu.Comparison Of Propofol And Thiopentone On Hemodynamic Responses To Modified Electroconvulsive Therapy: A Randomized Controlled Trial.KLE University, Belgaum, Karnataka. 652.2009.

[17] Loimer N, Hofmann P, Chaudry HR. Midazolam shortens seizure duration following electroconvulsive therapy. J Psychiatry Res 1992; 26: 97.

[18] Rasmussen KG, Jarvis MR, Zorumski CF. Ketamine anesthesia in ECT. Convulse Ther 1996; 12: 217.

[19] Kay B, Rolly G. IcI 32868, a new intravenous induction agent. Acta Anesthesia Belg 1977; 23: 303.

[20] Djordjevic B, Stojikovic MP. Cardiovascular effect of induction doses of intravenous anesthetics propofol. Etomidate and thiopental. Vojnosanit Pregl 1999; 56(1): 15-19.

[21] Gelissen HP, Epenma AH, Henning RH, et al. Inotropic effect of propofol, thiopental, midazolam. Etomidate and ketamine on isolated human atrial muscle. Anesthesia 1996; 84: 397-403.

[22] Komai H, Rusy BF. Effect on thiopental on care lease form sarcoplasmic reticulum in intact myocardium. Anesthesiology 1994; 87: 946-52.

[23] Roudy JJ, Andreev A, Leger P, et al. Peripheral vascular effects of thiopental and propofol in human with artificial heart. Anesthesiology 1991; 75: 32-42.

[24] Zaidi NA, Khan FA. Comparison of thiopentone sodium and propofol for ECT. J Pak Med Assoc 2000; 50(2): 60- 3.

[25] Boey WK, Lai FO. Comparison of propofol and thiopentone as anesthetic agents for ECT. Anesthesia 1990; 45(8): 23-8.

[26] Harti A, Hmamouchi B, Idali H, Barvou L. Anesthesia for ECT: propofol versus thiopental; Encephale 2001; 27(3): 217-21.

Citation: Hamid Kayalha et al., "Investigating the Effect of Drugs Thiopental Sodium and Propofol in Patients Undergoing Electroconvulsive Therapy, RCT”, International Journal of Research Studies in Medical and Health Sciences. 2020; 5(4): 24-28.

Copyright: (C) 2020 Hamid Kayalha et al., This is an open-access article distributed under the terms of the Creative Commons Attribution License, which permits unrestricted use, distribution, and reproduction in any medium, provided the original author and source are credited. 